eral non-governmental organizations, plays a vital role in the management of disasters in Nigeria. These roles vary from the prevention of disasters to the rehabilitation of victims of disasters. Investigations show that some of these disasters are the result of human inadequacies or poor management of government-owned facilities (e.g., refineries in the case of fire or illegal issuance of certificates of occupancy and approvals to builders who build to block drainages, in the case of flood-related disasters).

Some of the disasters resulted from poverty, such as pipeline vandalization. Pipelines are vandalized most frequently in the Niger Delta area in Nigeria, and, on several occasions, resulted in fires, which killed several people and destroyed properties worth millions of naira. This research analyzes the cost implications of some of these documented disasters in Nigeria during the pre-disaster, disaster, and post-disaster stages. The results reveal that during the predisaster phase, the cost is negligible, while the burden of the acute phase and the post-disaster phase is astronomically high.

In conclusion, if adequate funding is provided during the pre-disaster phase, perhaps the disaster can be completely avoided and the associated morbidity and mortality prevented. Keywords: cost analysis; disaster; management; Nigeria; pre-disaster; post-disaster; poverty; prevention Prebosp Disast Med 2005;20(2):s80-s81

\section{Injury Severity and Outcome Evaluation of Injured Patients of the Bam Earthquake}

M. Zare; A. Bidari; A. Hosseinnezbad; S. Hosseini;

N. Tavakoli; M. Mofidi; M. Yasinzadeb; A. Labbaf

Iran University Of Medical Sciences, Iran

Introduction: The Bam Earthquake occurred on 26 December 2003 in Iran, and the number of injured patients overwhelmed the nearby facilities in a short period of time. Patients were transferred to hospitals in other provinces the day after the disaster. Rasool-Akram Hospital, a Level-1 equivalent Trauma Center, located in the capital city of Tehran, accepted a large number of cases during the disaster. Objective: To evaluate the severity of trauma and outcome of earthquake victims in this referral hospital.

Methods: In this cohort study, all patients admitted to the hospital were examined during the course of hospitalization. The primary end point was either hospital discharge or patient death. The causes of mortality and the length of stay (LOS) were recorded. The revised trauma score (RTS), injury severity score (ISS), and trauma and injury severity score (TRISS) also were determined.

Results: Between 26 December 2003 and 03 January 2004, 180 patients injured in the earthquake were transferred to Rasool-Akram Hospital. Most cases (96.7\%) had a RTS score of 7.84. According to the ISS, 42 cases (23.3\%) had major trauma with an ISS of 16 , the average TRISS in the patients was $5.2 \pm 1.4$, and five patients (2.9\%) died during the course of stay. The expected mortality according to TRISS methodology was 2.65 (among 180 cases). The average LOS was $13.1 \pm 1.4$ days for survivors and average time to death in deceased cases was $10.0 \pm 6.2$ days.
Conclusion: Most transferred cases had minor injuries and the intra-hospital mortality was low, but the average length of stay was relatively high. In addition to trauma severity indexes, length of stay for patients injured by the earthquake may also be affected by medical complications, especially acute renal failure.

Keywords: Bam; earthquake; injury severity score (ISS); revised trauma score (RTS); trauma and injury severity score (TRISS) Prehosp Disast Med 2005;20(2):s81

Study of Respiratory Symptoms Related to Smoke Inhalation During the Sydney 2001-2002 Bushfire Event

G. Watkins

Australia

Introduction: On 25 December 2001, Sydney was shrouded in a thick cloud of black smoke fueled by up to 60 bushfires in and around the city caused by a combination of drought, hot weather, high winds, and arson. The smoke persisted for 13 days.

Objective: To obtain information about the health risks of bushfire smoke to enable the provision of appropriate public health advice, and to assist in bushfire smoke pollution management.

Methods: In early January 2002, a convenience sample was taken of 230 patients attending two Sydney emergency departments for any reason. These patients had all been exposed to bushfire smoke for nine days or more, and completed questionnaires regarding respiratory symptoms and exacerbation of pre-existing asthma or lung disease. The symptoms investigated were cough, shortness of breath, chest tightness, and wheezing. The same questionnaire was administered to a similar control group in January 2005. Seasonal variation in asthma and viral illnesses necessitated that the control group be obtained at the same time of year to reduce confounding variables. The original design was to obtain the control group in January 2003, however the study population experienced further bushfire smoke exposure, so the survey was postponed.

Results: A total of $51 \%$ percent of those surveyed during the bushfires reported one or more of the respiratory symptoms investigated.

Conclusions: A large proportion of the persons exposed to smoke have respiratory symptoms the persist well-beyond the period of exposure.

Keywords: asthma; bushfires; health risks; public health; respiratory symptoms; smoke inhalation; Sydney

Prehosp Disast Med 2005;20(2):s81

Psychosocial Care at Mass-Gathering Events: Integration into Emergency Medical Services during the EURO 2004 in Portugal

S. Miranda-Hyam, ${ }^{1}$ S. Cunba, ${ }^{1}$ J. Faria ${ }^{1}$ S. Rosado, ${ }^{1}$ M. Soares-Oliveira, ${ }^{2}$ N. Pereira ${ }^{1}$

1. Instituto Nacional de Emergência Médica (INEM), Portugal

2. Instituto Nacional de Emergência Médica (INEM), Puerto Rico

Background: The integration of psychologists with emergency medical services at mass-gathering events has not been well-described. 